\title{
Impactos socioambientales por la fabricación de ladrillos en Huancayo
}

\author{
Social and environmental impacts by making bricks in \\ Huancayo
}

Marisol Condori Apaza'

Universidad Nacional de Centro del Perú

\section{RESUMEN}

Objetivos: Identificar los impactos sociales y ambientales generados por el proceso de fabricación de ladrillos en las zonas de Palián y San Agustín de Cajas de la ciudad de Huancayo. Métodos: La investigación fue básica, nivel descriptivo, con diseño de investigación no experimental transversal y enfoque metodológico cuantitativo. Se aplicó un cuestionario a 80 trabajadores de las fábricas de ladrillos de las dos zonas de trabajo. Resultados: La fabricación de ladrillos generó impactos sociales tales como: perjuicios a la salud, 94\%; y generación de trabajo, 88\%; siendo la mayoría de trabajadores niños. Anteriormente el $64 \%$, se dedicaba a la agricultura; el 14\%, a la ganadería; actualmente, el $60 \%$ consideró que su estilo de vida ha cambiado desde la fabricación de ladrillos; el $64 \%$ de personas que trabajan en las fábricas manifestaron haber sufrido accidentes laborales. Respecto a los impactos ambientales generados por las actividades del proceso de fabricación de ladrillos en las zonas mencionadas; el 54\%, indicó que esta actividad es responsable de la baja fertilidad de las tierras agrícolas; el $81 \%$ consideró que antes de la fabricación de los ladrillos se respiraba aire menos contaminado; el 68\% afirmó que anteriormente a dicha actividad, consumían agua menos contaminada; el $86 \%$, manifestó que la fauna y la flora se alteró; el $61 \%$, consideró que el nivel de ruido se ha incrementado. Conclusiones: El proceso de fabricación de ladrillos en el distrito de San Agustín de Cajas y el anexo de Palian generó impactos sociales, fundamentalmente en

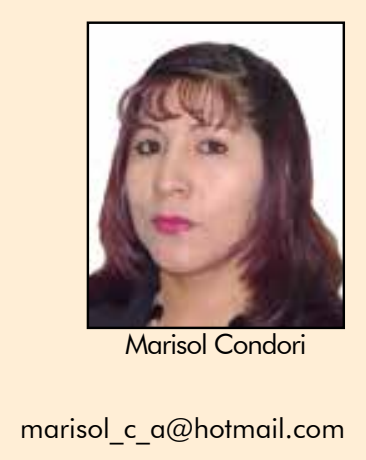

las actividades económicas, por lo tanto cambiando su estilo de vida; asimismo, afectó significativamente a diversos ecosistemas.

Palabras clave: Impacto social, ambiental, fabricación de ladrillos. 


\section{ABSTRACT}

Objectives: To identify the social and environmental impacts generated by the manufacturing process of bricks in Palian and San Agustin de Cajas in Huancayo city. Methods: Basic research, descriptive level, with no cross-experimental design research and quantitative methodological approach, since a structured 80 people (as) questionnaire was applied boxes and Annex Palian in the province of Huancayo. Results: Respondents indicate that the manufacturing process of bricks generates social impacts such as damage to health $(94 \%)$, generation of work ( $87 \%)$, with most workers children, changes in lifestyle, as previously $64 \%$ are engaged in agriculture, $14 \%$ for livestock, $60 \%$ consider their lifestyle before brickmaking was quiet, $64 \%$ of people working in the factories reported having been accidents. Regarding the environmental impacts generated by the activities of the manufacturing process of bricks in the above mentioned areas, the $54 \%$ indicates that this activity is responsible for the low fertility of agricultural land; $81 \%$ indicates that prior to bricks manufacturing air was less polluted, $68 \%$ believe that before this activity consumed less contaminated water $86 \%$ indicates that the fauna was altered; $86 \%$ indicates that the flora is altered, $61 \%$ consider that the noise level has increased. Conclusions: The process of making bricks in the district of San Agustin de Cajas and the annex Palian generates social and environmental impacts.

Keywords: Social impact, environmental, manufacturing bricks.

\section{INTRODUCCIÓN}

Las actividades económicas que se desarrollan en el Perú, son diversas, entre ellas formales e informales; desde la minería informal en la cual trabajan personas en situación social y económica vulnerables, hasta la fabricación artesanal de ladrillos que se desarrollan en diversos lugares de nuestro país; en esta oportunidad se investigó a la provincia de Huancayo, para lo cual se ha considerado los casos de ladrilleras artesanales del anexo de Palian y del distrito de San Agustín de Cajas.

La presente investigación es de importancia para las ciencias sociales, porque en el Perú, así como en la provincia de Huancayo, los impactos socio ambientales que generan las actividades económicas vienen afectando significativamente a diversos ecosistemas, así también afectan a los grupos sociales, en cuanto a su organización y unión. En tal sentido, la presente investigación tiene por finalidad abordar la problemática en mención desde un enfoque de la sociología del medio ambiente.

El Programa Regional Aire Limpio (1), realizó la investigación "Experiencias en el sector ladrillero artesanal en las ciudades de Arequipa y Cusco", cuyos resultados indican que, las ladrilleras de tamaños micro y pequeño aplican, en su mayoría, técnicas artesanales con hornos de baja eficiencia. Esta es compensada mediante el uso de combustibles de alto poder calorífico y bajo precio, tales como llantas usadas, plásticos, aceite quemado de vehículos, etcétera. Estos combustibles son, al mismo tiempo, altamente contaminantes, lo que convierte a la actividad ladrillera en fuente de contaminación que afecta la calidad del aire de las ciudades y poblaciones cercanas, la salud de sus habitantes, de los propios trabajadores y de sus familias. La actividad ladrillera artesanal se desenvuelve en un escenario especial caracterizado por: alta generación de contaminantes, informalidad, economía precaria, inseguridad en el trabajo y reducida capacidad de gestión.

Montoro (2) en su investigación "Gestión ambiental de la industria ladrillera en el distrito de Quilcas, provincia de Huancayo", muestra como resultados que la actividad ladrillera produce impactos en el ecosistema rural del distrito, usando de manera desmedida recursos como insumos para la elaboración de éstos como son los suelos y agua subterránea, reduciendo la capacidad productiva y afectando la disponibilidad del 
recurso hídrico, cuyo proceso de fabricación genera emisiones contaminantes al aire, lo cual ocasiona efectos negativos en la población, tales como: enfermedades respiratorias y generando la perturbación del paisaje rural.

Romo, Cervera y Córdova (3) en su "Estudio urbano-ambiental de las ladrilleras en el municipio de Juárez" evalúan la utilización de técnicas tradicionales para la elaboración del ladrillo, lo que provoca emisiones altamente contaminantes a la atmósfera. Esto tiene un fuerte impacto en el medio ambiente a nivel binacional, en la región Paso del Norte. Sin embargo, el contexto socioeconómico de los trabajadores de esta industria no permite cambios importantes; además, hace difícil cualquier iniciativa de mejorar y relocalizar los hornos ladrilleros.

Moreno y Soler (4) realizan una estimación de riesgos ambientales causados por la industria ladrillera en México, indicando que ésta es una actividad productiva que además de ser una fuente de empleo importante, genera un insumo básico para la industria de la construcción. Sin embargo, el proceso de producción emplea combustibles altamente contaminantes, que generan dioxinas y furanos, distintas especies de hidrocarburos, volúmenes masivos de partículas, monóxido de carbono, óxidos de azufre y de nitrógeno, contaminando la atmósfera, cuerpos de agua y suelo.

Ramirez (5) realizó el estudio del impacto ambiental en el proceso de elaboración de ladrillo en la comunidad Del Chote, Poza Rica, México; cuyos resultados demuestran que los impactos ambientales de este proceso fueron significativos e irreversibles, que afectaron a la tierra debido al uso de hornos afectando a los asentamientos humanos, así también al aire por los gases de salida de horno.

La presente investigación buscó responder a la pregunta ¿ Cuáles son los impactos socio ambientales generados por el proceso de fabricación de ladrillos en la provincia de Huancayo? Por ende, el objetivo de investigación fue identificar los impactos socio ambiental generado por el proceso de fabricación de ladrillos en la provincia de Huancayo. La hipótesis de investigación indica que los impactos sociales generados por el proceso de fabricación de ladrillos son: perjuicios a la salud de los pobladores, generación de empleo, cambios en el estilo de vida de la población, accidentes laborales

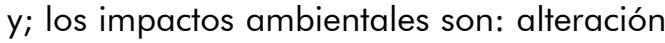
de las tierras agrícolas, contaminación del aire y agua, alteración de la fauna y flora, incremento de los niveles de ruido.

Con la presente investigación se contribuye al enriquecimiento de conocimientos de la sociología del medio ambiente.

\section{MATERIAL Y MÉTODOS}

El tipo de investigación fue básica, ya que, con los resultados hallados se contribuye al enriquecimiento del conocimiento respecto a los impactos generados por las actividades económicas informales y/o artesanales en nuestro país. La investigación considera el nivel descriptivo, ya que pretende describir los impactos sociales y ambientales que genera la fabricación artesanal de ladrillos en San Agustín de Cajas y Palian.

El diseño de la investigación es no experimental transversal descriptivo, con enfoque metodológico cuantitativo. La recolección de datos se realizó a través de un cuestionario estructurado a una muestra de 80 pobladores que se dedican a la fabricación de ladrillos del distrito de San Agustín de Cajas y el anexo de Palian, provincia de Huancayo, departamento de Junín.

\section{RESULTADOS}

Antes de presentar los resultados de la presente investigación, es necesario señalar 
Tabla $N^{\circ} 1$ : Impactos sociales generados por el proceso de fabricación de ladrillos

\begin{tabular}{lccccc}
\hline \multicolumn{1}{c}{ Impacto } & Frecuencia & $\%$ & Ho & Valor Z & Valor $\mathbf{p}$ \\
\hline Generación de trabajo & 70 & 88 & Ho: $\pi>0,5$ & $6,71^{* *}$ & 0,000 \\
Periuicios a la salud & 75 & 94 & Ho: $\pi>0,5$ & $7,83^{* *}$ & 0,000 \\
Vida intranquila & 48 & 60 & Ho: $\pi>0,33$ & $5,14^{* *}$ & 0,000 \\
$\begin{array}{l}\text { Sufrimiento de } \\
\text { accidentes laborales }\end{array}$ & 51 & 64 & Ho: $\pi>0,5$ & $2,47^{* *}$ & 0,007 \\
\hline Ho: Hipótesis nula; $\left.{ }^{* *}\right)$ Significativo al 1\% $(\mathrm{p}<0,01)$ & & & &
\end{tabular}

que, a partir de la década de los 90 , el impacto ambiental se aborda en términos más integrales, contemplando no sólo los efectos de los proyectos $-u$ actividades económicas- sobre el medio biofísico, sino también sobre el medio social y estudiando las interconexiones entre ambos (6). Así, la hipótesis de investigación indica que los impactos sociales generados por el proceso de fabricación de ladrillos son: perjuicios a la salud de los pobladores, generación de empleo, cambios en el estilo de vida de la población, accidentes laborales y; los impactos ambientales son: alteración de las tierras agrícolas, contaminación del aire y agua, alteración de la fauna y flora, incremento de los niveles de ruido. En tal sentido, a continuación se presentan los principales resultados hallados en el proceso de investigación.

En la tabla $N^{\circ} 1$, se aprecia que los impactos sociales generados por el proceso de fabricación de ladrillos en Palian y
San Agustín de Cajas - Huancayo son la generación de trabajo ( $p<0,01$ ), los perjuicios de la salud ( $p<0,01$ ), la vida intranquila $(p<0,01)$ y el sufrimiento de accidentes laborales por los trabajadores ( $p<0,01)$, tal como se evidencia con la prueba $Z$ de Gauss para una proporción en muestras grandes. El primero de los impactos es positivo, mientras que los tres últimos, negativos.

En la tabla $\mathrm{N}^{\circ} 2$ se observa que los impactos ambientales generados por el proceso de fabricación de ladrillos en Palián y San Agustín de Cajas - Huancayo son el aire contaminado que se respira $(p<0,01)$, el agua contaminada que se consume $(p<$ $0,01)$, la alteración de la fauna $(p<0,01)$, la alteración de la flora $(p<0,01)$ y el incremento del ruido $(p<0,05)$, tal como se evidencia con la prueba $Z$ de Gauss para una proporción en muestras grandes. Estos impactos son negativos. La alteración de la fertilidad de los suelos resulta ser

Tabla № 2: Impactos ambientales generados por el proceso de fabricación de ladrillos

\begin{tabular}{lccccc}
\hline \multicolumn{1}{c}{ Impacto } & Frecuencia & $\%$ & Ho & Valor Z & Valor $p$ \\
\hline $\begin{array}{l}\text { Alteración de la fertilidad } \\
\text { de los suelos }\end{array}$ & 43 & 54 & Ho: $\pi>0,5$ & 0,67 & 0,251 \\
$\begin{array}{l}\text { Aire contaminado que se } \\
\text { respira }\end{array}$ & 65 & 81 & Ho: $\pi>0,33$ & $9,18^{* *}$ & 0,000 \\
$\begin{array}{l}\text { Agua contaminada que } \\
\text { se consume }\end{array}$ & 54 & 68 & Ho: $\pi>0,33$ & $6,56^{* *}$ & 0,000 \\
$\begin{array}{l}\text { Alteración de la fauna } \\
\text { Alteración de la flora }\end{array}$ & 69 & 86 & Ho: $\pi>0,5$ & $6,48^{* *}$ & 0,000 \\
\begin{tabular}{l} 
Incremento del ruido \\
\hline
\end{tabular} & 49 & 61 & Ho: $\pi>0,5$ & $6,48^{* *}$ & 0,000 \\
\end{tabular}

Ho: Hipótesis nula; $\left({ }^{* *}\right)$ Significativo al $1 \%(p<0,01)$; $\left({ }^{*}\right)$ Significativo al $5 \%(p<0,05)$ 


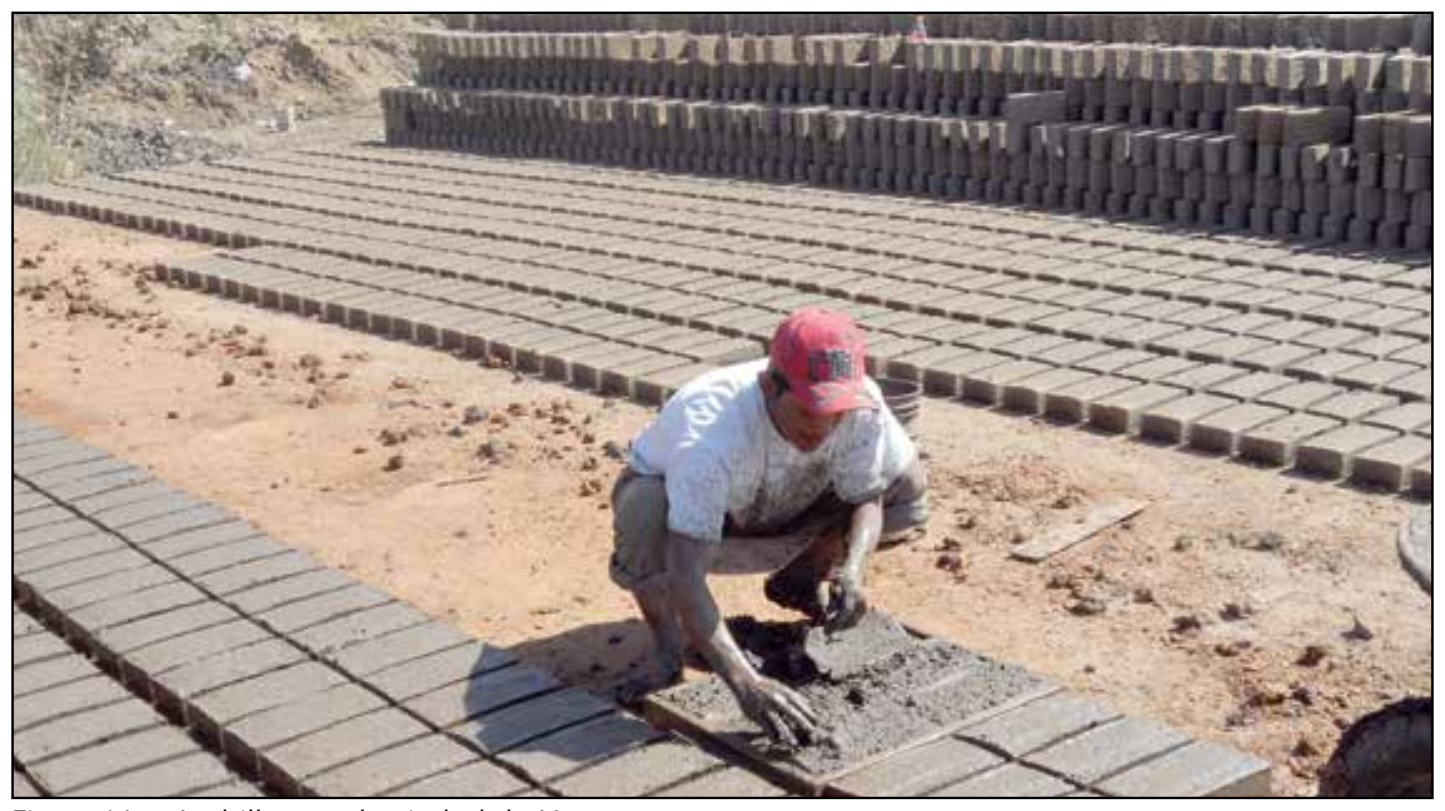

Figura $\mathrm{N}^{\circ}$ 1: Ladrillera en la ciudad de Huancayo.

no significativo $(p>0,05)$ a la luz de la prueba $Z$ de Gauss para una proporción en muestras grandes.

\section{DISCUSIÓN}

En la década de las noventa cuestiones internacionales como locales configuran un escenario particular para la introducción de la evaluación de impacto ambiental en los países en desarrollo y si bien aún se está lejos del ideal, la dimensión social en función del contexto internacional hoy tiene otro lugar en este tema. El paradigma fiscalista sigue aún vigente, pero frente a él se encuentra el paradigma sociológico que incorpora el concepto de vulnerabilidad de la población frente al análisis de programas, políticas, proyectos, normativas $y / 0$ actividades económicas diversas. Esto genera a su vez la incorporación de especialistas de las Ciencias Sociales en el tratamiento de las cuestiones ambientales con una visión sistémica que da lugar a otro marco de acción que va más allá de las técnicas de evaluación ambiental de proyectos y que hacen al tratamiento integral de la problemática ambiental (6).
La evaluación y los impactos ambientales, deben ser analizados desde diversos enfoques, y coincidiendo con Soriano (7) estos son el territorial, demográfico, económico y sociocultural, ya que cualquier actividad económica o ejecución de proyecto, genera impactos de diversa índole. En esta oportunidad, abordaremos los impactos sociales y ambientales que genera la fábrica artesanal de ladrillos en el distrito de san Agustín de Cajas y el anexo de Palian de la provincia de Huancayo.

Respecto a los impactos sociales generados por esta actividad, los resultados muestran que el $94 \%$ de encuestados(as) considera haber sido perjudicado en su salud. Similar situación halló Montoro (2) indicando que el proceso de fabricación de ladrillos en Quilcas ocasiona efectos negativos en la población tales como enfermedades respiratorias. Asimismo el PRAL (1) considera que esta actividad es fuente de contaminación y afecta la salud de sus habitantes, de los propios trabajadores y de sus familias.

Sin embargo, un impacto considerado positivo por los encuestados es que, la actividad ladrillera genera trabajo (88\%), aspecto también considerado por Moreno y Soler (4) quienes manifiestan que, es una actividad productiva que 
además de ser una fuente de empleos importante, genera un insumo básico para la industria de la construcción. Sin embargo, se debe dejar claro, tal como manifiestan Gonzales, Perea, Ojeda y Matamoros (8) que esta actividad artesanal tradicionalmente ha sido desarrollada por personas de estratos sociales marginados que encuentran en este oficio una forma de autoempleo medianamente estable, pero lamentablemente muy mal remunerada, poco valorada y menos reconocida por la sociedad.

Por otro lado, esta actividad generó cambios en el estilo de vida de la población, ya que anteriormente el $64 \%$ se dedicaba a la agricultura, el $14 \%$ a la ganadería. Al respecto, Codes (9) señala que en El Algarrobal el modo de vida agrícola prácticamente ha desaparecido.

Otro dato, nos revela que, el $64 \%$ de personas que trabajan en las fábricas de San Agustín de Cajas y Palian, manifestaron haber sufrido accidentes laborales; lo cual, desde la perspectiva de Noriega (10) son riesgos laborales derivados de los elementos del proceso laboral que potencialmente pueden crear daños a la salud y pueden clasificarse en riesgos físicos, químicos, biológicos y mecánicos. Los accidentes laborales se ubican dentro de los riesgos mecánicos que, de acuerdo a los resultados de la presente investigación se asemejan a los hallazgos de Gonzales, Perea, Ojeda y Matamoros (8), quienes demuestran que, en lo que respecta a los riesgos mecánicos, los que más comúnmente se presentan son los golpes contusos, "machucones", laceraciones y caídas. Estos se presentan a lo largo del proceso de trabajo.

Respecto a los impactos ambientales generados por las actividades del proceso de fabricación de ladrillos en las zonas estudiadas; el $54 \%$ indica que esta actividad es responsable de la baja fertilidad de las tierras agrícolas; el $81 \%$ indica que antes se respiraba aire menos contaminado; el $68 \%$ considera que antes consumía agua menos contaminada. Similar aseveración realiza Montoro (2) dando a conocer que en Quilcas las ladrilleras usan de manera desmedida recursos como insumos para la elaboración de éstos como son los suelos y agua subterránea, reduciendo la capacidad productiva y afectando la disponibilidad del recurso hídrico, cuyo proceso de fabricación genera emisiones contaminantes al aire, a esta aseveración se suma Ramírez (5) quien manifiesta que el proceso de fabricación de ladrillos en la comunidad Del Chote, México, genera impactos significativos e irreversibles al suelo y aire. En el mismo sentido, el PRAL (1) considera que la actividad ladrillera es fuente de contaminación que afecta la calidad del aire de las ciudades y poblaciones cercanas. Sumándose a estos resultados Moreno y Soler (4) demuestran que, el proceso de producción de ladrillos emplea combustibles altamente contaminantes, que generan dioxinas y furanos, distintas especies de hidrocarburos, volúmenes masivos de partículas, monóxido de carbono, óxidos de azufre y de nitrógeno, contaminando la atmósfera, cuerpos de agua y suelo.

Finalmente, $86 \%$ indica que la flora de las zonas se alteró. Al respecto Codes y Robledo (9) consideran que, la existencia de la ladrillera produce un impacto negativo elevado sobre la vegetación.

\section{REFERENCIAS BIBLIOGRÁFICAS}

1. Programa Regional Aire Limpio. Experiencias en el sector ladrillero artesanal en las ciudades de Arequipa y Cusco Perú: COSUDE; 2008.

2. Montoro Y. Gestión Ambiental de la industrialadrilleraeneldistritodeQuilcas, Provincia de Huancayo. [Tesis Doctoral]. Huancayo: Universidad Nacional del Centro del Perú; (Recuperado el 13 de diciembre del 2013) En: http:// ymeldamontorozamora.wordpress. com/proyecto-de-investigacion/

3. Romo ML, Cervera LE, y Córdova G. Estudio urbano-ambiental de las 
ladrilleras en el municipio de Juárez. México: Universidad Autónoma de Baja California; 2004. (Recuperado el 30 de noviembre del 2013). En: http://www. redalyc.org/articulo.oa?id $=53050901$

4. Moreno P, Soler FI. Estimación de riesgos ambientales causados por la industria Ladrillera. División de estudios de Posgrado, Facultad de Ingeniería de la UNAM, 2003. (recuperado el 06 de marzo del 2014) En: http://www. bvsde.paho.org/bvsaidis/puertorico29/ moreno.pdf

5. Ramirez BE. Estudio del impacto ambiental en el proceso de elaboración de ladrillo en la comunidad Del Chote. Tesis para obtener el Título Profesional de Ingeniero Ambiental de la facultad de Ciencias Químicas de la Universidad Veracruzana, México 2011. /Recuperado el 03 de abril del 2014) En: http://cdigital.uv.mx/ bitstream/123456789/29499/1/ RamirezBarbosa.pdf

6. Irigalba AC, Ełxaleku AI, Echavarren JM. La evaluación de impacto ambiental. Recopilación, análisis y punto de vista crítico desde la perspectiva sociológica. (Recuperado el 01 de diciembre del 2013) En: http://rua.ua.es/dspace/ bitstream/10045/2725/9/cap9.pdf

7. Soriano $M$. Evaluación de impacto ambiental. Legislación y metodología. Cuadernos de Ciencia y técnicas ambientales. Serie Medio Ambiente. Instituto de estudios Baleáricos; 1992.

8. Gonzáles N, Perea C, Ojeda S, Matamoros M. El oficio del ladrillero. Sus riesgos y exigencias. Chiguaga: Facultad de Enfermería y Nutriología, Instituto de Bellas Artes/Universidad Autónoma de Chihuahua, 2008. (Recuperado el 15 de diciembre del 2013) En: http:// www.uach.mx/extension_y_difusion/ synthesis/2008/11/10/ladrillero.pdf

9. Codes MI, Robledo SB. Impacto ambiental de las ladrilleras en el Algarrobal, departamento de las Heras, Mendoza, Argentina: Facultad de Filosofía y Letras. SeCyTP. UNCuyo; 2002.

10. Noriega, M. Organización laboral, exigencias y enfermedad. Investigación sobre salud de los trabajadores. Washington: Organización Panamericana de la Salud; 1993. 